\title{
Engagement no trabalho em profissionais de programas de aprimoramento profissional em saúde ${ }^{1}$
}

\author{
Elizangela Gianini Gonsalez ${ }^{a}$, Luciano Garcia Lourençáa ${ }^{b}$, Priscila Regina Teixeira $^{a}$, \\ Daniela Salvagni Rotta ${ }^{a}$, Cláudia Eli Gazetta ${ }^{a}$, Denise Beretta ${ }^{a}$, Maria Helena Pinto ${ }^{a}$

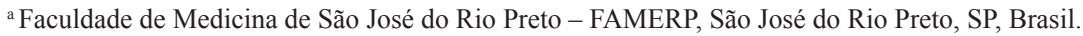 \\ bUniversidade Federal do Rio Grande - FURG, Rio Grande, RS, Brasil.
}

\begin{abstract}
Resumo: Objetivo: Este estudo avaliou os níveis de engagement no trabalho em profissionais de programas de aprimoramento e aperfeiçoamento profissional em saúde. Método: Realizou-se um estudo transversal com 82 profissionais de saúde dos programas de aprimoramento e aperfeiçoamento de uma instituição pública do interior paulista, utilizando-se a Utrech Work Engagement Scale (UWES), um questionário autoaplicável composto de 17 itens de autoavaliação em três dimensões: vigor, dedicação e absorção. Os escores foram calculados conforme modelo estatístico proposto no Manual Preliminar UWES. Resultados: Os níveis de engagement foram muito altos na dimensão vigor, altos na dimensão dedicação e no escore geral e médio na dimensão absorção para $71,61 \%$, $58,03 \%, 53,75 \%$ e $51,22 \%$ dos profissionais, respectivamente. Os profissionais apresentaram relação positiva com o trabalho, são responsáveis, motivados e dedicados ao trabalho. Conclusão: O estudo reforça a importância de avaliar os aspectos positivos da relação entre profissionais e ambiente laboral, contribuindo para fortalecer os programas de aprimoramento e aperfeiçoamento profissionais.
\end{abstract}

Palavras-chave: Desenvolvimento de Pessoal, Profissional de Saúde, Internato e Residência.

\section{Work engagement in employees at professional improvement programs in health}

\begin{abstract}
Objective: This study evaluated the levels of engagement at work in enhancement programs and professionals training in health. Method: A cross-sectional study with 82 health professionals enhancement programs and improvement of a public institution in the State of São Paulo, using the Utrech Work Engagement Scale (UWES), a self-administered questionnaire composed of seventeen self-assessment items in three dimensions: vigor, dedication and absorption. The scores were calculated according to the statistical model proposed in the Preliminary Manual UWES. Results: Engagement levels were too high on the force, high dedication and dimension in general score, and medium in size to $71.61 \%$ absorption, $58.03 \%, 53.75 \%$ and $51.22 \%$ of workers, respectively. The professionals present positive relationship with the work; they are responsible, motivated and dedicated to the job and to the patients. Conclusion: Reinforces the importance of studies that evaluate positive aspects of the relationship between professionals and working environment, contributing to strengthen the programs of improvement, advancing the profile of professionals into the labour market.
\end{abstract}

Keywords: Staff Development, Health Personnel, Internship and Residency.

\footnotetext{
Autor para correspondência: Luciano Garcia Lourenção, Escola de Enfermagem, Universidade Federal do Rio Grande, Rua General Osório, s/n, $4^{\circ}$ piso, Centro, CEP 96201-900, Rio Grande, RS, Brasil, e-mail: luciano.famerp@gmail.com

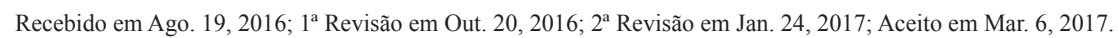




\section{Introdução}

Os programas de aprimoramento profissional em saúde são categorias de pós-graduação lato sensu, desenvolvidos na modalidade de formação em serviço sob supervisão, mantidos com recursos do governo do estado de São Paulo (SÃO PAULO, 2015; LOURENÇÃO; MOSCARDINI; SOLER, 2013). Esses programas são ofertados por instituiçóes vinculadas ao Sistema Único de Saúde (SUS) e têm o objetivo de capacitar os profissionais a exercerem a profissão com mais qualidade, atendendo à demanda dos usuários do sistema público de saúde (SÃO PAULO, 2015).

Seguindo o modelo dos Programas de Aprimoramento Profissional da Secretaria de Estado da Saúde de São Paulo, a Faculdade de Medicina de São José do Rio Preto (Famerp), instituição em regime especial de autarquia estadual, em parceria com a Fundação Faculdade Regional de Medicina de São José do Rio Preto (Funfarme), oferece o Programa de Aperfeiçoamento Profissional em Saúde, mantido com recursos próprios (SÃO PAULO, 2015; FACULDADE..., 2016).

Sabe-se que os profissionais inseridos nesses programas de formação em serviço estão constantemente sob grande pressão e, por isso, são vulneráveis a alteraçôes psicológicas, como ansiedade e depressão, que podem comprometer a qualidade de vida, o bem-estar e a satisfação dos profissionais e, consequentemente, prejudicam a qualidade da assistência à saúde dos usuários dos serviços de saúde. Fatores como a sobrecarga de trabalho, o contato frequente com a dor e o sofrimento, o lidar com a intimidade corporal e emocional, o atendimento de pacientes terminais, a atuação em pacientes críticos, em risco de morte ou não aderentes ao tratamento ou cronicamente deprimidos, além da convivência com as incertezas e limitaçóes do conhecimento profissional e do sistema assistencial (CARVALHO et al., 2013; LOURENÇÃO; MOSCARDINI; SOLER, 2013; JIMÉNEZ; SOCORRO; ALIÑO, 2010; LOURENÇÃO; MOSCARDINI; SOLER, 2010), estão presentes e não podem ser menosprezados.

Contudo, apesar das exigências e do desgaste gerado vivido durante o processo de formação, alguns profissionais podem náo apresentar sinais e sintomas de exaustáo. Ao contrário, apresentam um estado cognitivo positivo de realização, demonstrando retirar prazer do trabalho árduo e suas exigências. Esses profissionais apresentam engagement no trabalho, um estado cognitivo positivo de realizaçáo e bem-estar com o trabalho que se caracteriza por três dimensóes: vigor, dedicação e absorção (BASSO-MACHADO;
PORTO-MARTINS, 2013; PORTO-MARTINS; BASSO-MACHADO; BENEVIDES-PEREIRA, 2013; ANGST; BENEVIDES-PEREIRA; PORTO-MARTINS, 2009).

O termo engagement foi conceituado pela primeira vez em 1990, por Willian A. Kahn (1990). Trata-se de uma concepção recente, compreendida inicialmente como

[...] aproveitamento dos membros da organização de seus próprios papéis de trabalho: no engagement, as pessoas utilizam e se expressam a si mesmas física, cognitiva, emocional e mentalmente durante o desenvolvimento de seus papéis [...] (ANGST; BENEVIDES-PEREIRA; PORTOMARTINS, 2009, p. 694).

Portanto, trabalhadores com bons níveis de engagement apresentam-se mais satisfeitos com a profissão, possuem maior bem-estar e demonstram intençóes de permanecer no local de trabalho (SANTOS, 2011).

À luz da psicologia positiva, o engagement ganha força à medida que contribui para revelar aspectos psicossociais relevantes da relaçáo dos profissionais com o trabalho (TEIXEIRA et al., 2017; PORTO-MARTINS; BASSO-MACHADO, 2010). Além disso, pode causar um impacto positivo na empresa/organização ao favorecer a adoção de medidas de proteçáo para a saúde dos trabalhadores (ORTIZ; JARAMILLO, 2012).

Estudos sobre engagement são recentes no Brasil, especialmente com profissionais de saúde. Uma pesquisa realizada com residentes médicos de pediatria apontou bons índices de engagement entre esses profissionais, especialmente no domínio dedicação, mostrando que os médicos residentes encontram-se entusiasmados e orgulhosos com o trabalho (TEIXEIRA et al., 2017). Para os autores, o desenvolvimento de estudos que abordem esse constructo entre os profissionais em formação poderá trazer benefícios para a organização e o aprimoramento do processo de trabalhos nesses programas de formação.

Basso-Machado, Porto-Martins e Amorim (2012) corroboram a importância do desenvolvimento de estudos sobre engagement no Brasil, que favoreçam a compreensão dos aspectos que interferem no desenvolvimento desse constructo, dada a relevância dos fatores positivos na relação dos profissionais com o trabalho.

Portanto, avaliar o engagement no trabalho de aprimorandos e aperfeiçoandos da área da saúde torna-se relevante para conhecer as relaçôes desses profissionais com o ambiente de trabalho e obter 
informaçóes que contribuam para o fortalecimento do processo de formação oferecido e com a qualidade do atendimento prestado aos pacientes.

Ante o exposto, este estudo objetivou avaliar os níveis de engagement no trabalho dos profissionais matriculados nos programas de aprimoramento e aperfeiçoamento de uma instituição pública do interior do estado de São Paulo.

\section{Método}

Trata-se de um estudo transversal, de base populacional, realizado com todos os profissionais matriculados nos Programas de Aprimoramento e Aperfeiçoamento Profissional de uma instituição de ensino do interior do estado de São Paulo. Ambos são programas de pós-graduaçāo lato sensu multiprofissionais, que objetivam a formação profissional em serviço, sob supervisão, desenvolvidos com jornada semanal de 40 (quarenta) horas, constituindo-se de atividades teóricas (20\%) e práticas (80\%), conforme determina a legislação (FACULDADE..., 2016).

O Programa de Aprimoramento Profissional é um programa de bolsas mantido pela Secretaria de Estado da Saúde de São Paulo (SES/SP), ofertado por diversas instituiçôes de ensino do estado e está direcionado a profissionais das diferentes áreas da saúde, especialmente recém-formados, com exceção da medicina, mantido pela Secretaria da Saúde do Estado de Sáo Paulo e oferecido por diversas instituições vinculadas ao SUS (SÃO PAULO, 2015).

O Programa de Aperfeiçoamento Profissional está estruturado nos mesmos moldes do Programa de Aprimoramento da SES/SP. É o único programa dessa categoria ofertado pela Faculdade de Medicina de São José do Rio Preto, com as bolsas de estudo custeadas pela Fundação Faculdade Regional de Medicina de São José do Rio Preto (Funfarme) (FACULDADE..., 2016).

O critério de inclusão no estudo foi ser aprimorando ou aperfeiçoando matriculado no programa. Foram excluídos aqueles que estavam afastados das atividades profissionais por qualquer natureza durante o período de coleta dos dados e os profissionais do programa de aperfeiçoamento que cursavam o segundo ano de formação, modalidade que era ofertada exclusivamente por esse programa na época do estudo.

Os dados foram coletados no período de novembro de 2013 a fevereiro de 2014. Os pesquisadores não possuíam vínculo com os programas, evitando possíveis vieses de influência nas respostas.
Para a coleta dos dados, foram utilizados dois instrumentos: um questionário elaborado pelos autores, contendo dados pessoais, como idade, sexo, estado civil, escolaridade, renda familiar, e se o profissional está satisfeito com o programa/processo de formaçáo ou se, em algum momento, pensou em desistir dos Programas de Aprimoramento/Aperfeiçoamento Profissional, e a Utrech Work Engagement Scale (UWES), um questionário autoaplicável composto de 17 itens de autoavaliaçấo com três dimensôes: vigor, dedicação e absorção, além de um escore geral do constructo (PORTO-MARTINS; BASSO-MACHADO; BENEVIDES-PEREIRA, 2013; SALANOVA; SCHAUFELI, 2009; BAKKER et al., 2008). Os profissionais matriculados nos programas, que aceitaram participar do estudo, responderam aos questionários no início de uma aula teórica, com a prévia permissão do professor responsável.

O vigor corresponde a elevados níveis de energia e resiliência, vontade de investir esforços, não se fadigar com facilidade e persistir diante das dificuldades. Profissionais com escores altos nessa dimensão possuem muita energia para trabalhar, enquanto os que apresentam escores baixos apresentam menos energia (PORTO-MARTINS; BASSO-MACHADO; BENEVIDES-PEREIRA, 2013; BAKKER; ALBRECHT; LEITER, 2011; ANGST; BENEVIDES-PEREIRA; PORTO-MARTINS, 2009; SALANOVA; SCHAUFELI, 2009).

A dedicação se refere ao significado do trabalho para o profissional, que se sente entusiasmado e orgulhoso, e se encontra inspirado e desafiado pela atividade laboral. Os profissionais com altos escores no domínio dedicação veem o trabalho como uma experiência significativa, inspiradora e desafiadora. Aqueles que apresentam baixos escores não encontram significado no trabalho, que não lhes desperta interesse nem entusiasmo (PORTO-MARTINS; BASSO-MACHADO; BENEVIDES-PEREIRA, 2013; BAKKER; ALBRECHT; LEITER, 2011; ANGST; BENEVIDES-PEREIRA; PORTO-MARTINS, 2009; SALANOVA; SCHAUFELI, 2009).

A absorção está relacionada ao nível de imersão e ao envolvimento do profissional com o trabalho. Profissionais com altos níveis de absorção apresentam dificuldades para se desapegar do trabalho, não percebem o tempo passar e se esquecem de tudo ao seu redor (PORTO-MARTINS; BASSO-MACHADO; BENEVIDES-PEREIRA, 2013; BAKKER; ALBRECHT; LEITER, 2011; ANGST; BENEVIDES-PEREIRA; PORTO-MARTINS, 2009; SALANOVA; SCHAUFELI, 2009). 
Para análise do engagement no trabalho, os cálculos dos escores foram realizados conforme modelo estatístico proposto no Manual Preliminar UWES (ANGST; BENEVIDES-PEREIRA; PORTO-MARTINS, 2009), apresentando-se valores mínimo, máximo, mediana, média ( \pm desvio-padrão) e intervalo de confiança de $95 \%$ para cada dimensão da UWES. Realizou-se, então, a interpretação dos valores obtidos, segundo o modelo indicado no Manual Preliminar UWES, conforme apresentado na Tabela 1.

A análise dos dados foi realizada com o programa Statistical Package for Social Sciences (SPSS), versão 17.0.

Por se tratar de dois programas estruturados e implementados nos mesmos moldes, com atuação conjunta dos profissionais no mesmo espaço e sob o mesmo processo de trabalho, não foram analisadas diferenças nas respostas entre os profissionais dos diferentes programas.

As comparaçôes foram feitas pelo teste qui-quadrado, considerando significante valor-p inferior ou igual a 0,05 .

O estudo foi aprovado pelo Comitê de Ética em Pesquisa da instituição, com Parecer n ${ }^{\circ} 319.203$, de 11 de junho de 2013.

\section{Resultados}

Participaram do estudo 82 profissionais de diferentes categorias, dos quais 31 eram aprimorandos e 51, aperfeiçoandos.

Conforme apresentado na Tabela 2, observou-se maior prevalência de profissionais do sexo feminino. A faixa etária variou de 20 a 35 anos, sendo a idade mediana de 25 anos. Em relaçáo ao estado civil, observou-se que a maioria era solteira. Quarenta e cinco profissionais $(54,88 \%)$ tinham renda familiar de dois a cinco salários mínimos e $28,05 \%$, de seis a dez salários mínimos.

Destaca-se, ainda, que 49 (59,75\%) profissionais referiram estar satisfeitos com o programa no qual

Tabela 1. Modelo para interpretação dos valores dos escores médios do estudo.

\begin{tabular}{ll}
\hline Classificação & $\begin{array}{c}\text { Frequência dos sentimentos em } \\
\text { relação ao trabalho (questões da } \\
\text { UWES) }\end{array}$ \\
\hline Muito baixo & 0 a $0,99=1$ (algumas vezes por ano) \\
\hline Baixo & 1 a $1,99=2$ (uma vez ou menos por mês) \\
\hline Médio & 2 a $2,99=3$ (algumas vezes por mês) \\
\cline { 2 - 2 } 3 a $3,99=4$ (uma vez por semana) \\
\hline Alto & 4 a $4,99=5$ (algumas vezes por semana) \\
\hline Muito alto & 5 a $6=6$ (todos os dias) \\
\hline
\end{tabular}

estavam matriculados, embora 50 (60,98\%) já tivessem pensado em desistir do programa.

A Tabela 3 apresenta os resultados da avaliação dos níveis de engagement no trabalho, segundo a distribuição dos valores mínimo, máximo, mediana, média ( \pm desvio-padráo) e intervalo de confiança de $95 \%$, segundo as dimensôes da UWES: vigor, dedicação, absorção e escore geral. A média das dimensóes variou de 3,76 a 4,51 e os desvios-padrão variaram entre 0,95 e 1,07. A dimensão Dedicação obteve a maior média $(4,51 \pm 1,06)$, classificada como alta, assim como o escore geral obteve média de 4,04 (dp: $\pm 0,95)$. As dimensóes Vigor e Absorção obtiveram

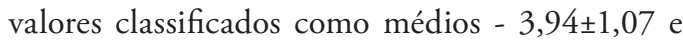
$3,76 \pm 1,03$, respectivamente.

$\mathrm{Na}$ análise da confiabilidade, os valores do Coeficiente Alfa de Cronbach variaram de 0,693 a 0,900, conforme mostra a Tabela 4 . Nessa tabela também está apresentada a distribuição do número de profissionais em cada nível de classificação do engagement no trabalho. Destacam-se os percentuais de profissionais que apresentaram níveis de engagement altos e muito altos nas dimensôes vigor $(58,03 \%$; $\mathrm{n}=47)$, dedicação $(71,61 \% ; \mathrm{n}=58)$ e escore geral $(53,75 \% ; n=43)$. A dimensão absorção apresentou $51,22 \%(\mathrm{n}=42)$ dos profissionais com nível de engagement médio.

Os níveis de engagement no trabalho, segundo o desejo de desistir e a satisfaçáo com os programas de aprimoramento e aperfeiçoamento, são apresentados na Tabela 5. Observou-se que as médias obtidas foram maiores entre os profissionais que nunca pensaram em desistir e os que se referiram satisfeitos com os programas para todas as dimensóes da UWES. Houve diferença estatisticamente significante das médias entre os dois grupos, para todas as dimensões avaliadas.

Em relação aos que pensaram ou não em desistir do programa, com exceção da dimensão dedicação, em que a média obtida foi alta para ambos os grupos (4,32 e 4,84, respectivamente), para as demais dimensôes os níveis foram médios para os que já haviam pensado em desistir do programa e altos para aqueles que nunca haviam pensado em desistir. Sobre a satisfação com o programa, os resultados foram semelhantes: na dimensão dedicação, níveis altos tanto para os satisfeitos quanto para os insatisfeitos (4,88 e 4,00, respectivamente); nas dimensóes vigor, absorção e escore geral, os níveis foram altos para os satisfeitos (4,32, 4,16 e 4,41, respectivamente) e médios para os insatisfeitos $(3,38,3,25$ e 3,49, respectivamente). 
Tabela 2. Distribuição das características dos profissionais matriculados nos programas de aprimoramento e aperfeiçoamento profissional da Faculdade de Medicina de São José do Rio Preto. São José do Rio Preto, 2013-2014.

\begin{tabular}{|c|c|c|c|}
\hline & & $\mathrm{n}$ & $\%$ \\
\hline \multirow{8}{*}{ Categoria profissional } & Enfermagem & 21 & 25,61 \\
\hline & Farmácia & 02 & 2,44 \\
\hline & Fisioterapia & 25 & 30,49 \\
\hline & Fonoaudiologia & 06 & 7,32 \\
\hline & Nutrição & 02 & 2,44 \\
\hline & Psicologia & 12 & 14,63 \\
\hline & Serviço Social & 08 & 9,76 \\
\hline & Terapia Ocupacional & 06 & 7,32 \\
\hline \multirow{2}{*}{ Sexo } & Masculino & 12 & 14,63 \\
\hline & Feminino & 70 & 85,37 \\
\hline \multirow{4}{*}{ Faixa etária } & 20 a 25 anos & 39 & 47,56 \\
\hline & 26 a 30 anos & 29 & 35,37 \\
\hline & 31 a 35 anos & 01 & 1,22 \\
\hline & Não respondeu & 01 & 1,22 \\
\hline \multirow{3}{*}{ Estado civil } & Casado & 04 & 4,88 \\
\hline & Solteiro & 74 & 90,24 \\
\hline & Outros & 04 & 4,88 \\
\hline \multirow{5}{*}{ Renda familiar (salários mínimos) } & Até 1 & 07 & 8,54 \\
\hline & 2 a 5 & 45 & 54,88 \\
\hline & 6 a 10 & 23 & 28,05 \\
\hline & Mais de 10 & 06 & 7,32 \\
\hline & Não respondeu & 01 & 1,22 \\
\hline \multirow{2}{*}{ Satisfeito com o programa } & Sim & 49 & 59,75 \\
\hline & Não & 33 & 40,24 \\
\hline \multirow{2}{*}{ Pensou em desistir do programa } & Sim & 50 & 60,98 \\
\hline & Não & 32 & 39,02 \\
\hline
\end{tabular}

Tabela 3. Distribuição dos valores mínimo, máximo, mediana, média ( \pm desvio-padrão) e intervalo de confiança de 95\%, para as dimensões da Utrecht Work Engagment Scale (UWES), segundo a avaliação dos profissionais matriculados nos programas de aprimoramento e aperfeiçoamento da Faculdade de Medicina de São José do Rio Preto. São José do Rio Preto, 2013-2014.

\begin{tabular}{lcccccccc}
\hline $\begin{array}{l}\text { Dimensões da } \\
\text { UWES }\end{array}$ & N & Mín & Máx & Md & Média \pm DP & IC (95\%) & Interpretação & Valor-p \\
\hline Vigor & 81 & 0,33 & 5,67 & 4,16 & $3,94 \pm 1,07$ & $3,70-4,17$ & Médio & 0,127 \\
Dedicação & 81 & 2,20 & 6 & 4,60 & $4,51 \pm 1,06$ & $4,27-4,74$ & Alta & 0,076 \\
Absorção & 82 & 1,67 & 5,67 & 3,83 & $3,76 \pm 1,03$ & $3,53-3,99$ & Média & 0,128 \\
Escore geral & 80 & 1,59 & 5,71 & 4,11 & $4,04 \pm 0,95$ & $3,83-4,25$ & Alto & 0,147 \\
\hline
\end{tabular}

Mín: mínimo; Máx: máximo; Md: mediana; DP: desvio-padrão; IC95\%: intervalo de confiança de 95\%.

Tabela 4. Distribuição do coeficiente de confiabilidade, escore médio e número de profissionais, segundo a classificação do nível de engagement para as dimensões da Utrecht Work Engagment Scale (UWES). São José do Rio Preto, 2013-2014.

\begin{tabular}{|c|c|c|c|c|c|c|c|c|c|c|c|c|}
\hline \multirow{3}{*}{$\begin{array}{l}\text { Dimensões da } \\
\text { UWES }\end{array}$} & \multirow{3}{*}{$\begin{array}{l}\text { Alfa de } \\
\text { Cronbach }\end{array}$} & \multirow{3}{*}{$\begin{array}{l}\text { Escore } \\
\text { médio }\end{array}$} & \multicolumn{10}{|c|}{ Classificação do nível de engagement no trabalho } \\
\hline & & & \multicolumn{2}{|c|}{ Muito baixo } & \multicolumn{2}{|c|}{ Baixo } & \multicolumn{2}{|c|}{ Médio } & \multicolumn{2}{|c|}{ Alto } & \multicolumn{2}{|c|}{ Muito alto } \\
\hline & & & 4 & $\%$ & $\mathbf{N}$ & - & $\mathbf{N}$ & $\%$ & $\mathbf{N}$ & 0 & $\mathbf{N}$ & $\%$ \\
\hline & & & 01 & 1,23 & 0 & 1, & 32 & & 3( & & 7 & 20.99 \\
\hline & & & & - & - & - & 23 & & 24 & 3 & 34 & 41, \\
\hline s & 0,693 & 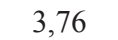 & 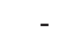 & - & 01 & 1,2 & 42 & 51,22 & 27 & 3 & 12 & 14 , \\
\hline Escore geral & 0,900 & 4,04 & - & _ & 02 & 2,5 & 35 & 43,75 & 27 & 33,75 & 16 & 20,0 \\
\hline
\end{tabular}


Tabela 5. Distribuição das médias ( \pm desvio-padrão) para as escalas da Utrecht Work Engagment Scale (UWES), segundo desejo de desistir e satisfação referida pelos profissionais matriculados nos programas de aprimoramento e aperfeiçoamento da Faculdade de Medicina de São José do Rio Preto. São José do Rio Preto, 2013-2014.

\begin{tabular}{|c|c|c|c|c|c|}
\hline Dimensão da UWES & Pensou em desistir & $\mathbf{N}$ & Média $\pm D P$ & Interpretação & Valor-p (t-test) \\
\hline \multirow{2}{*}{ Vigor } & Sim & 50 & $3,69 \pm 1,03$ & Médio & \multirow{2}{*}{0,001} \\
\hline & Não & 31 & $4,36 \pm 1,01$ & Alto & \\
\hline \multirow{2}{*}{ Dedicação } & Sim & 50 & $4,32 \pm 1,08$ & Alta & \multirow{2}{*}{0,002} \\
\hline & Não & 31 & $4,84 \pm 0,94$ & Alta & \\
\hline \multirow{2}{*}{ Absorção } & Sim & 50 & $3,5 \pm 0,97$ & Média & \multirow{2}{*}{0,001} \\
\hline & Não & 32 & $4,25 \pm 0,98$ & Alta & \\
\hline \multirow{2}{*}{ Escore geral } & Sim & 50 & $3,81 \pm 0,90$ & Médio & \multirow{2}{*}{0,004} \\
\hline & Não & 30 & $4,43 \pm 0,92$ & Alto & \\
\hline Dimensão da UWES & Satisfação com o programa & $\mathbf{N}$ & Média $\pm D P$ & Interpretação & Valor-p (t-test) \\
\hline \multirow{2}{*}{ Vigor } & Sim & 49 & $4,32 \pm 0,93$ & Alto & \multirow{2}{*}{$<0,001$} \\
\hline & Não & 32 & $3,38 \pm 1,04$ & Média & \\
\hline \multirow{2}{*}{ Dedicação } & Sim & 48 & $4,88 \pm 0,85$ & Alta & \multirow{2}{*}{$<0,001$} \\
\hline & Não & 33 & $4,00 \pm 1,11$ & Alta & \\
\hline \multirow{2}{*}{ Absorção } & Sim & 49 & $4,16 \pm 0,97$ & Alta & \multirow{2}{*}{$<0,001$} \\
\hline & Não & 33 & $3,25 \pm 0,89$ & Média & \\
\hline \multirow{2}{*}{ Escore geral } & Sim & 48 & $4,41 \pm 0,84$ & Alto & \multirow{2}{*}{$<0,001$} \\
\hline & Não & 32 & $3,49 \pm 0,84$ & Médio & \\
\hline
\end{tabular}

\section{Discussão}

O trabalho pode causar efeitos positivos e negativos sobre o profissional, dependendo da sua relação laboral com o ambiente (BASSO-MACHADO; PORTO-MARTINS; AMORIM, 2012).

Historicamente, diversos pesquisadores têm se debruçado sobre os aspectos negativos do trabalho sobre os profissionais de saúde, inclusive com profissionais inseridos em programas de formação em serviço, especialmente residentes médicos (CARVALHO et al., 2013; LOURENÇÃO; MOSCARDINI; SOLER, 2013; JIMÉNEZ; SOCORRO; ALIÑO, 2010; LOURENÇÃO; MOSCARDINI; SOLER, 2010; NOGUEIRA-MARTINS, 2010; ANGST; BENEVIDES-PEREIRA; PORTO-MARTINS, 2009). Entretanto, poucos estudos abordando as fortalezas e o bom funcionamento do ser humano no trabalho têm sido desenvolvidos, principalmente no Brasil (PORTO-MARTINS; BASSO-MACHADO, 2010).

A análise dos dados sociodemográficos deste estudo mostrou distribuição dos profissionais em diferentes categorias profissionais, com predomínio de fisioterapeutas $(30,49 \%)$ e enfermeiros (25,31\%); prevalência de profissionais do sexo feminino (85,37\%), na faixa etária de 20 a 30 anos (82,93\%), solteiros $(90,24 \%)$ e com renda entre dois e cinco salários mínimos (54,88\%).
A distribuição dos profissionais deste estudo em diferentes categorias profissionais é característica inerente ao Programa de Aprimoramento Profissional mantido pelo governo do estado de São Paulo, por meio da Secretaria de Estado da Saúde, ofertado em diversas categorias profissionais não médicas (SÃO PAULO, 2015).

A prevalência de profissionais de fisioterapia e enfermagem na população de estudo é inerente à distribuição do número de vagas entre as profissóes e às características do hospital do estudo. Trata-se de um hospital-escola de alta complexidade que presta serviços especializados de referência para mais de 100 municípios da regiáo noroeste do estado de Sáo Paulo e oferece, anualmente, 113 vagas, distribuídas entre os Programas de Aprimoramento (55 vagas) e Aperfeiçoamento (58 vagas) Profissional em Saúde. Destas, 34 vagas são para especialidades na fisioterapia e 30 vagas para diferentes especialidades na enfermagem (FACULDADE..., 2016).

Ao mesmo tempo que este estudo apontou que houve uma maioria de profissionais que referiram estar satisfeitos com o programa de formação $(59,75 \%)$, apresentou um grande percentual de profissionais que, em algum momento, já havia pensado em desistir do processo (60,98\%). Resultados semelhantes foram observados entre residentes multiprofissionais de uma universidade pública brasileira (GOULART et al., 2012). 
Embora os profissionais estejam satisfeitos com o processo de formaçãa, diversas causas de desgaste emocional são vivenciadas ao longo desse processo, como intensa jornada de trabalho, precariedade de alguns serviços, falta de materiais, dificuldades de relacionamento com pacientes, familiares e membros da equipe de saúde, insegurança e medo de errar, dificuldade de conciliar trabalho e estudos, entre outras (VELHO et al., 2012; ASAIAG et al., 2010; LOURENÇÃO; MOSCARDINI; SOLER, 2010). A presença desses fatores gera desgaste emocional nos profissionais, podendo levar ao desejo de deixar o programa, apesar da satisfação com o processo de formaçáo/aprendizagem.

Os profissionais avaliados apresentaram escores que variaram entre médios e altos $(3,76$ a 4,51) para o engagement no trabalho, evidenciando uma relação positiva com o trabalho que desempenham ao longo da formação no aprimoramento/aperfeiçoamento, além de níveis de energia elevados e a identificação com o trabalho na profissão escolhida (BAKKER; LEITER, 2010).

Observou-se, ainda, grande percentual de profissionais com níveis altos e muito altos de engagement no trabalho nas dimensôes vigor (58,03\% dos profissionais com escores alto e muito alto), dedicação ( $71,61 \%$ com escores alto e muito alto) e no escore geral $(53,75 \%$ com escores alto e muito alto).

O vigor representa um componente comportamentalenergético da relação do profissional com o trabalho e caracteriza-se por altos níveis de energia, persistência, desejo de esforçar-se no trabalho e resiliência mental. A dedicação é o componente emocional caracterizado pela concentração plena na realização do trabalho, inspiração, orgulho, desafio, objetivo, significado e entusiasmo (VELHO et al., 2012; ASAIAG et al., 2010; BAKKER; LEITER, 2010; SALANOVA; SCHAUFELI, 2009). Portanto, os profissionais estudados mostraram uma relação muito boa com o trabalho.

Além disso, mais de $50 \%$ dos profissionais apresentaram escores médios na dimensão absorção. A absorção é um componente cognitivo caracterizado pela concentração e pela felicidade na realização do trabalho, sensação de que o tempo passa muito rápido e dificuldade para desligar-se do trabalho (VELHO et al., 2012; ASAIAG et al., 2010; BAKKER; LEITER, 2010; SALANOVA; SCHAUFELI, 2009).

Resultados semelhantes foram encontrados com profissionais de saúde de unidades de atenção primária em saúde de Lisboa, em Portugal, que apresentaram níveis de engagement variando de 4,67 a 4,88. Os escores observados nas dimensóes vigor, dedicação e absorção foram, respectivamente, de 4,85, 5,00 e 4,80 para enfermeiros e 4,83, 4,68 e 4,79 para médicos, níveis de engagement considerados altos (SANTOS, 2011).

Corroborando, ainda, com os achados do presente estudo, ao avaliarem a qualidade de vida de profissionais de programas de aprimoramento e aperfeiçoamento, autores concluíram que os profissionais se encontravam satisfeitos com a qualidade de vida, a vida e a saúde, apresentavam bom nível de independência e boa estrutura espiritual/religiosa (LOURENÇÃO; MOSCARDINI; SOLER, 2013).

Esses resultados evidenciam, em uma análise global, uma relaçáo positiva dos profissionais estudados com o trabalho em que, como consequência, podem apresentar-se mais motivados, com mais facilidade de interagir com pacientes, familiares e colegas, mais dispostos e criativos, com maior rendimento nos estudos e na produtividade, além da diminuição de erros e redução do absenteísmo.

Embora os resultados demonstrem que os profissionais estudados apresentam boas condições emocionais (dedicação), a redução observada nas dimensôes vigor, absorção e escore geral entre os profissionais que já pensaram em desistir ou que se referiram insatisfeitos com o programa evidencia que o desgaste emocional comumente vivenciado ao longo do processo de aprimoramento reflete na relação do profissional com o trabalho, comprometendo os níveis de energia e a identificação com o trabalho (engagement). Como consequência, pode haver comprometimento na aprendizagem do profissional, redução do desempenho profissional, perda da qualidade de vida e risco de adoecimento, culminando com queda na qualidade da assistência prestada aos usuários.

Assim, apesar de a legislação pertinente ao Programa de Aprimoramento Profissional não exigir a implementaçáo de serviços de apoio psicológico aos profissionais, a presença desse serviço e oferta de apoio aos profissionais se torna fundamental para o melhor aproveitamento desse sistema de capacitação profissional (SILVA et al., 2011), garantindo qualidade na formação de profissionais para o SUS.

Por fim, destaca-se que, para que haja um engagement verdadeiro entre os profissionais inseridos em programas de aprimoramento/aperfeiçoamento, é importante que os supervisores e os profissionais em formaçáo construam uma relação positiva, de confiança, respeito e mutuamente benéfica, de modo que ambos acreditem e se esforcem para promover 
o crescimento dentro do sistema. Nesse sentido, autores destacam que os níveis de engagement podem ser melhorados com base em alguns fatores relacionados ao trabalho, como carga de trabalho, controle, recompensa, comunidade, justiça e valores (BAKKER; ALBRECHT; LEITER, 2011).

\section{Conclusão}

Os resultados deste estudo mostraram profissionais com níveis altos de engagement nas dimensóes dedicação e no escore geral e médios nas dimensões absorção e vigor. Os profissionais que se referiram satisfeitos e os que nunca pensaram em desistir do programa apresentaram maior engagement.

Esses resultados sugerem que os profissionais avaliados apresentam uma relação positiva com o trabalho, são responsáveis e encontram-se motivados e dedicados ao trabalho. Reforçam, portanto, a importância de estudos que avaliem os aspectos positivos da relação entre os profissionais e o ambiente laboral, contribuindo para o fortalecimento dos programas de aprimoramento e a consequente melhoria do perfil dos profissionais inseridos no mercado de trabalho.

O fato de os profissionais estudados pertencerem a uma única instituição representa uma limitação para este estudo. Além disso, as variaçóes relacionadas aos processos de trabalho nos diferentes setores hospitalares não foram investigadas.

Sugere-se, portanto, que outros estudos sejam realizados, ampliando o quantitativo de profissionais e as instituiçóes avaliadas, analisando as particularidades de cada especialidade, permitindo a comparação de grupos originários de regióes e realidades distintas, bem como a discussão das especificidades do processo de trabalho das diferentes especialidades profissionais, com o pressuposto de que diferenças significativas possam ser analisadas, contribuindo para o avanço do conhecimento sobre o engagement no trabalho entre esses profissionais.

\section{Referências}

ANGST, R.; BENEVIDES-PEREIRA, A. M. T.; PORTO-MARTINS, P. C. Utrecht Work Engagement Scale. Curitiba: GEPEB, 2009.

ASAIAG, P. E. et al. Avaliaçấo da qualidade de vida, sonolência diurna e burnout em Médicos Residentes. Revista Brasileira de Educação Médica, Rio de Janeiro, v. 34, n. 3, p. 422-429, 2010.

BAKKER, A. B. et al. Work engagement: an emerging concept in occupational health psychology. Work and Stress, United States, v. 22, n. 3, p. 187-200, 2008.
BAKKER, A. B.; ALBRECHT, S. L.; LEITER, M. P. Work engagement: further reflections on the state of play. European Journal of Work and Organizational Psychology, Hove, v. 20, n. 1, p. 74-88, 2011.

BAKKER, A. B.; LEITER, M. P. Where to go from here: integration and future research on work engagement. In: BAKKER, A. B.; LEITER, M. P. (Org.). Work engagement: a handbook of essential theory and research. New York: Psychology Press, 2010. p. 181-196.

BASSO-MACHADO, P. G.; PORTO-MARTINS, P. C. Condiçóes organizacionais enquanto terceiras variáveis entre burnout e engagement. Revista da Sociedade de Psicologia do Rio Grande do Sul, Porto Alegre, v. 13, n. 1, p. 35-44, 2013.

BASSO-MACHADO, P. G.; PORTO-MARTINS, P. C.; AMORIM, C. Engagement no trabalho entre profissionais da educaçáo. Revista Intersaberes, Curitiba, v. 7, n. 13, p. 193-214, 2012.

CARVALHO, C. N. et al. Prevalência e fatores associados aos transtornos mentais comuns em residentes médicos e da área multiprofissional. Jornal Brasileiro de Psiquiatria, Rio de Janeiro, v. 62, n. 1, p. 38-45, 2013.

FACULDADE DE MEDICINA DE SÃO JOSÉ DO RIO PRETO - FAMERP. Comissão de Aprimoramento e Aperfeiçoamento da Funfarme e Famerp. Processo Seletivo 2016. São José do Rio Preto, 2016. Disponível em: <http://www.famerp.br/novoportal/index.php/processo-seletivo-coaprimo\#.Vo_hYPkrLDc>. Acesso em: 08 jan. 2016.

GOULART, C. T. et al. Perfil sociodemográfico e acadêmico dos residentes multiprofissionais de uma universidade pública. Revista Rene, Natal, v. 13, n. 1, p. 178-186, 2012.

JIMÉNEZ, O. G.; SOCORRO, M. P. M.; ALIÑO, J. J. L. Psicopatología y factores de riesgo durante la residencia. Actas Españolas de Psiquiatría, Madrid, v. 38, n. 2, p. 65-71, 2010.

KAHN, W. A. Psychological conditions of personal engagement and disengagement at work. Academy of $\mathrm{Ma}$ nagement Journal, New York, v. 33, n. 4, p. 692-724, 1990.

LOURENÇÃO, L. G.; MOSCARDINI, A. C.; SOLER, Z. A. S. G. Health and quality of life of medical residents. Revista da Associação Médica Brasileira, São Paulo, v. 56, n. 1, p. 81-91, 2010.

LOURENÇÃO, L. G.; MOSCARDINI, A. C.; SOLER, Z. A. S. G. Qualidade de vida de residentes náo médicos. Revista de Enfermagem UFPE on line, Recife, v. 7, n. 11, p. 6336-6345, 2013.

NOGUEIRA-MARTINS, L. A. Qualidade de vida dos médicos residentes: revisão de estudos brasileiros. Cadernos da ABEM, Rio de Janeiro, v. 6, n. 1, p. 12-18, 2010.

ORTIZ, F. A.; JARAMILLO, V. A. Factores de riesgo psicosocial y compromiso (engagement) com el trabajo 
en una organización del sector salud de la ciudad de Cali, Colombia. Acta Colombiana de Psicologia, Colômbia, v. 16, n. 1, p. 43-56, 2012.

PORTO-MARTINS, P. C.; BASSO-MACHADO, P. G. Engagement en el trabajo. Studies and Research Psychology, Rio de Janeiro, v. 10, n. 3, p. 972-976, 2010.

PORTO-MARTINS, P. C.; BASSO-MACHADO, P. G.; BENEVIDES-PEREIRA, A. M.T. Engagement no trabalho: uma discussão teórica. Fractal: Revista de Psicologia, Niterói, v. 25, n. 3, p. 629-644, 2013.

SALANOVA, M.; SCHAUFELI, W. B. El engagement en el trabajo. Madrid: Alianza, 2009.

SANTOS, F. M. F. C. A satisfação profissional e o engagement nos profissionais de saúde do ACES Oeste Sul. 2011. 80 f. Dissertação (Mestrado em Gestão dos Serviços de Saúde) - Instituto Universitário de Lisboa, Lisboa, 2011. Disponível em: <https://repositorio.iscte-iul.pt/bitstream/10071/4175/1/Tese\%20ACES.pdf>. Acesso em: 6 jan. 2016.
SÃO PAULO. Governo do Estado. Secretaria de Estado da Saúde. Fundação do Desenvolvimento Administrativo - FUNDAP. Programa de Aprimoramento Profissional (PAP): manual de orientaçóes técnicas e administrativas. São Paulo: FUNDAP, 2015. Disponível em: <http:// pap.fundap.sp.gov.br/arquivos/manual_pap2015.pdf>. Acesso em: 15 nov. 2015.

SILVA, G. C. C. et al. A importância do apoio psicológico ao médico residente e especializando em radiologia e diagnóstico por imagem. Radiologia Brasileira, São Paulo, v. 44, n. 2, p. 81-84, 2011.

TEIXEIRA, P. R. et al. Engagement no trabalho em residentes médicos de pediatria. Revista Brasileira de Educação Médica, Rio de Janeiro, v. 41, n. 1, p. 126-133, 2017.

VELHO, M. T. A. C. et al. Residência médica em um hospital universitário: a visão dos residentes. Revista Brasileira de Educação Médica, Rio de Janeiro, v. 36, n. 3, p. 351-357, 2012.

\section{Contribuição dos Autores}

Luciano contribuiu para a concepção do projeto, análise e interpretação dos dados e redação do artigo. Cláudia, Maria Helena e Denise contribuíram para a redação do artigo, revisão crítica relevante do conteúdo intelectual e aprovação final da versão a ser publicada. Priscila Regina, Elizangela e Daniela contribuíram com a coleta, análise e interpretação dos dados e redação do artigo. Todos os autores aprovaram a versão final do texto.

\section{Fonte de Financiamento}

Faculdade de Medicina de São José do Rio Preto - FAMERP, Programa de Bolsas de Auxílio a Pesquisa 2013-2014. Protocolo n. 001-003966/2013.

\section{Notas}

${ }^{1}$ Artigo resultante da dissertação de mestrado intitulada Ansiedade, depressão e engagement no trabalho em aprimorandos e aperfeiçoandos da Faculdade de Medicina de São José do Rio Preto (SP), defendida em fevereiro de 2016, no Programa de Pós-Graduação em Enfermagem, sob orientação da Profa. Dra. Maria Helena Pinto e coorientação do Prof. Dr. Luciano Garcia Lourenção. Por se tratar de pesquisa envolvendo seres humanos, o estudo foi submetido à avaliação do Comitê de Ética em Pesquisa (CEP-Famerp/SP) e aprovado com parecer favorável à sua realização, Parecer n. 319.203, de 6 de junho de 2013. 